\title{
Comment on "Two-dimensional third-and fifth-order nonlinear evolution equations for shallow water waves with surface tension" [Nonlinear Dyn, doi:10.1007/s11071-017-3938-7]
}

\author{
Piotr Rozmej@ • Anna Karczewska
}

Received: 19 May 2021 / Accepted: 3 July 2021 / Published online: 31 July 2021

(C) The Author(s) 2021

\begin{abstract}
The authors of the paper "Two-dimensional third-and fifth-order nonlinear evolution equations for shallow water waves with surface tension" Fokou et al. (Nonlinear Dyn 91:1177-1189, 2018) claim that they derived the equation which generalizes the KdV equation to two space dimensions both in first and second order in small parameters. Moreover, they claim to obtain soliton solution to the derived first-order $(2+1)$ dimensional equation. The equation has been obtained by applying the perturbation method Burde (J Phys A: Math Theor 46:075501, 2013) for small parameters of the same order. The results, if correct, would be significant. In this comment, it is shown that the derivation presented in Fokou et al. (Nonlinear Dyn 91:11771189,2018 ) is inconsistent because it violates fundamental properties of the velocity potential. Therefore, the results, particularly the new evolution equation and the dynamics that it describes, bear no relation to the problem under consideration.
\end{abstract}

Keywords Shallow water waves $\cdot$ Boussinesq system of equations $\cdot$ Soliton solutions

P. Rozmej ( $\square)$

Faculty of Physics and Astronomy, University of Zielona

Góra, Szafrana 4a, 65-246 Zielona Góra, Poland

e-mail: P.Rozmej@if.uz.zgora.pl

A. Karczewska

Faculty of Mathematics, Computer Science and

Econometrics, University of Zielona Góra, Szafrana 4a,

65-246 Zielona Góra, Poland

e-mail: A.Karczewska@wmie.uz.zgora.pl

\section{Outline of the method}

The authors of [1] consider the model of inviscid and incompressible fluid which motion is irrotational in a container with a flat bottom. In dimensional variables, the set of hydrodynamical equations has the following form:

$\phi_{x x}+\phi_{y y}+\phi_{z z}=0, \quad$ in the volume,

$\phi_{z}-\left(\eta_{x} \phi_{x}+\eta_{y} \phi_{y}+\eta_{t}\right)=0, \quad$ at the surface,

$\phi_{t}+\frac{1}{2}\left(\phi_{x}^{2}+\phi_{y}^{2}+\phi_{z}^{2}\right)+g \eta=0, \quad$ at the surface, (3)

$\phi_{z}=0$ at the bottom.

Here $\phi(x, y, z, t)$ denotes the velocity potential, $\eta(x, y, t)$ anand denotes the surface function and $g$ is the gravitational acceleration. Indexes denote partial derivatives, i.e. $\phi_{x x} \equiv \frac{\partial^{2} \phi}{\partial x^{2}}$, and so on. The authors take into account surface tension terms, as well. In this note, we neglect these terms since their presence or absence does not change the source of errors made in [1].

Next, the authors introduce a standard scaling to dimensionless variables (different in $x$ - and $y$ direction)

$\tilde{x}=x / L, \quad \tilde{y}=y / y_{0}, \quad \tilde{z}=z / h_{0}, \quad \tilde{t}=t / t_{0}$,

$\tilde{\eta}=\eta / A, \quad \tilde{\phi}=\phi /\left(L \frac{A}{h_{0}} \sqrt{g h_{0}}\right)$,

where $A$ is the amplitude of surface distortions from equilibrium shape (flat surface), $h_{0}$ is average fluid depth, $L$ is the average wavelength (in $x$-direction), 
and $y_{0}$ is a wavelength in $y$-direction. In general, $y_{0}$ should be of the same order as $L$, but not necessarily equal. Notation $t_{0}=L / \sqrt{g h_{0}}$ is not explained in the paper.

Then, the set (1)-(4) takes in scaled variables the following form (tildas are now dropped):

$$
\begin{aligned}
& \beta \phi_{x x}+\gamma \phi_{y y}+\phi_{z z}=0, \\
& \eta_{t}+\alpha\left(\eta_{x} \phi_{x}+\frac{\gamma}{\beta} \eta_{y} \phi_{y}\right)-\frac{1}{\beta} \phi_{z}=0, \\
& \quad \text { for } z=1+\alpha \eta, \\
& \phi_{t}+\frac{1}{2} \alpha\left(\phi_{x}^{2}+\frac{\gamma}{\beta} \phi_{y}^{2}+\frac{1}{\beta} \phi_{z}^{2}\right)+\eta=0, \\
& \quad \text { for } z=1+\alpha \eta, \\
& \phi_{z}=0 \text { for } z=0 .
\end{aligned}
$$

As usual, small parameters are defined as follows:

$\alpha=A / h_{0}, \quad \beta=\left(h_{0} / l\right)^{2}$ and $\gamma=\left(h_{0} / y_{0}\right)^{2}$.

\section{Details of calculations in [1] limited to first order}

Next, the authors assume $\gamma=\beta$ and write erroneous formula [1, Eq. (11)] for the velocity potential

$$
\begin{gathered}
\phi=\sum_{\substack{m=0 \\
2_{n=0}^{2(m+n)}}}^{N} \frac{(-1)^{m+n} \beta^{m+n} z^{2(m+n)}}{(2 m) !(2 n) !} \\
\frac{\partial^{2 m} \partial y^{2 n}}{\partial x^{2 m}} f(x, y, t) .
\end{gathered}
$$

In the following, the authors limit their considerations to the Boussinesq equations up to second order in small parameters. Then, it is enough to use the explicit form of the potential (11) up to third order (due to terms $\frac{1}{\beta} \phi_{z}$ in (8) and $\frac{1}{\beta} \phi_{z}^{2}$ in (9) the velocity potential should be valid up to one order higher than the Boussinesq equations)

$$
\begin{aligned}
\phi=f- & \frac{\beta z^{2}}{2}\left(f_{x x}+f_{y y}\right)+\frac{\beta^{2} z^{4}}{24} \\
& \times\left(f_{x x x x}+6 f_{x x y y}+f_{y y y y}\right)-\frac{\beta^{3} z^{6}}{720} \\
& \times\left(f_{6 x}+15 f_{4 x 2 y}+15 f_{2 x 4 y}+f_{6 y}\right) .
\end{aligned}
$$

The fact that formulas (11)-(12) are wrong is easy to check by a direct substitution to the Laplace equation (7). With the above form of the velocity potential, the authors obtained the set of Boussinesq's equations in the form (here surface tension is neglected and only terms of first order are retained)

$$
\begin{aligned}
\eta_{t} & +f_{x x}+f_{y y}+\alpha\left(f_{x} \eta_{x}+f_{y} \eta_{y}+\eta\left(f_{2 x}+f_{2 y}\right)\right) \\
& -\frac{1}{6} \beta\left(f_{x x x x}+6 f_{x x y y}+f_{y y y y}\right)=0 \\
\eta_{x} & +f_{x t}+\alpha\left(f_{x} f_{x x}+f_{y} f_{x y}\right) \\
& -\frac{1}{2} \beta\left(f_{x y y t}+f_{x x x t}\right)=0 .
\end{aligned}
$$

Next, the authors insert the velocity potential into Eqs. (8) and (9) retaining terms up to second order in $\alpha, \varepsilon=$ $\beta$. They introduce the following notation

$u=f_{x}, \quad v=f_{y}$.

With the above notation Boussinesq's equations (13)(14) can be formulated as [1, Eq. (12)]- [1, Eq. (13)], that is as

$$
\begin{aligned}
& \eta_{t}+u_{x}+v_{y}+\alpha\left(u \eta_{x}+v \eta_{y}+\eta\left(u_{x}+v_{y}\right)\right) \\
& -\frac{1}{6} \varepsilon\left(u_{x x x}+3 u_{x y y}+3 v_{x x y}+v_{y y y}\right)=0, \\
& \eta_{x}+u_{t}+\alpha\left(u u_{x}+v v_{x}\right)-\frac{1}{2} \varepsilon\left(v_{x y t}+u_{x x t}\right)=0 .
\end{aligned}
$$

In the following, the authors apply the perturbative approach described in detail by Burde and Sergyeyev [2] and next extended in [3] to more complicated cases. In this method, one begins from zeroth-order solutions, then uses their properties in the calculation of corrections of the first order, and so on. In zeroth order eqs. [1, Eq. (12)]- [1, Eq. (13)] reduce to

$\eta_{t}+u_{x}+v_{y}=0, \quad$ and $\quad \eta_{x}+u_{t}=0$,

which have solutions $u=\eta, v=a(x, t)$ with $\eta_{t}=$ $-\eta_{x}, u_{t}=-u_{x}$. The authors of [1] take constant $v=$ $a$, which is a particular solution to $v_{y}=0$.

Looking for the solution in first-order approximation, one introduces corrections of the first order to the equations (18) and requires that the Boussinesq's equations become compatible in this order (what means that these two equations become equivalent). The authors look for first-order solutions in the form

$u=\eta+\alpha B(x, y, t)+\varepsilon C(x, y, t)$,

$v=a \quad$ (constant).

Inserting first-order corrections (19)-(20) into (16)(17), retaining only terms up to first order, and using 
properties $B_{t}=-B_{x}, C_{t}=-C_{x}$ the authors obtained simple differential equations for the correction functions $B$ and $C$. Here, we cite the formulas from [1]. More detailed derivation, with the correct velocity potential formula, is presented in Sect. 3. These equations, after integration give the formulas [1, Eq. (23)][1, Eq. (24)]. These formulas read as

$$
B=-\frac{1}{4} \eta^{2}-\frac{1}{2} a \int \eta_{y} d y, \quad C=\frac{1}{4} \eta_{y y}+\frac{1}{3} \eta_{x x} .
$$

So, in first-order approximation $u$ becomes (in [1, Eq. (25)] terms at $\alpha$ and $\varepsilon$ are incorrectly positioned)

$$
\begin{aligned}
u=\eta & +\alpha\left(-\frac{1}{4} \eta^{2}-\frac{1}{2} a \int \eta_{y} d y\right) \\
& +\varepsilon\left(\frac{1}{4} \eta_{y y}+\frac{1}{3} \eta_{x x}\right) .
\end{aligned}
$$

Insertion of $u$, given by the above expression into [1, Eq. (12)] and [1, Eq. (13)] leads in both cases to the same equation [1, Eq. (26)], that is

$$
\begin{gathered}
\eta_{t}+\eta_{x}+\alpha\left(\frac{3}{2} \eta \eta_{x}+\frac{1}{2} a \eta_{y}\right) \\
+\varepsilon\left(\frac{1}{6} \eta_{x x x}-\frac{1}{4} \eta_{x y y}\right)=0 .
\end{gathered}
$$

In other words, equation [1, Eq. (25)] supply compatibility of Boussinesq's equations and therefore may supply the correct $(2+1)$-dimensional evolution equation in the first order. On this basis, the authors extend the derivations to second order in small parameters and claim to obtain the (2+1)-dimensional fifth-order evolution equation [1, Eq. (32)]. They also claim to find the analytic solution to the equation [1, Eq. (26)] and discuss its properties.

We do not comment here the derivation of secondorder equation nor the authors' solutions to the firstorder equation because already first-order wave equation is incorrect. The details of the argumentation that the equation (22), that is, [1, Eq. (26)], is wrong are presented in Sect. 4.

\section{Details of correct calculations in first-order perturbation approach}

The correct formula for the velocity potential fulfilling (1) has the following form

$$
\phi(x, z, t)=\sum_{m=0}^{\infty} \frac{(-1)^{m}}{(2 m) !} z^{2 m}\left(\beta \partial_{x x}+\gamma \partial_{y y}\right)^{m} f(x, y, t) .
$$

For $\gamma=\beta$, the explicit form of this velocity potential up to third order in small parameters reads as

$$
\begin{aligned}
\phi=f- & \frac{\beta z^{2}}{2}\left(f_{x x}+f_{x y}\right)+\frac{\beta^{2} z^{4}}{24} \\
& \times\left(f_{x x x x}+2 f_{x x y y}+f_{y y y y}\right)-\frac{\beta^{3} z^{6}}{720} \\
& \times\left(f_{6 x}+3 f_{4 x 2 y}+3 f_{2 x 4 y}+f_{6 y}\right) .
\end{aligned}
$$

The correct formula for the velocity potential (23) and (24) differs from that used by the authors ((11) and (12)) by values of coefficients in front of mixed $x y$ derivatives.

With the correct formula (24), one obtains the following Boussinesq's equations. From (8), limiting to first order, one gets

$$
\begin{gathered}
\eta_{t}+f_{x x}+f_{y y}+\alpha\left(f_{x} \eta_{x}+f_{y} \eta_{y}+\eta\left(f_{2 x}+f_{2 y}\right)\right) \\
-\frac{1}{6} \epsilon\left(f_{x x x x}+2 f_{x x y y}+f_{y y y y}\right)=0 .
\end{gathered}
$$

The correct result from (9) in first order (after differentiation over $x$ ) is

$$
\begin{gathered}
\eta_{x}+f_{x t}+\alpha\left(f_{y} f_{x y}+f_{x} f_{2 x}\right) \\
-\frac{1}{2} \epsilon\left(f_{x y y t}+f_{x x x t}\right)=0 .
\end{gathered}
$$

In variables $u, v(15)$, equations (25)-(26) become

$$
\begin{aligned}
& \eta_{t}+u_{x}+v_{y}+\alpha\left(\eta_{x} u+\eta_{y} v+\eta\left(u_{x}+v_{y}\right)\right) \\
& -\frac{1}{6} \epsilon\left(u_{x x x}+2 u_{x y y}+v_{y y y}\right)=0, \\
& \eta_{x}+u_{t}+\alpha\left(u u_{x}+v v_{x}\right)-\frac{1}{2} \epsilon\left(u_{y y t}+u_{x x t}\right)=0 .
\end{aligned}
$$


Equations (25) and consequently (27) differ from equations (13) and (14) obtained from incorrect velocity potential (11) by the factor in front of the term $f_{x x y y}$.

Let us follow the authors' approach with the correct equations (27)-(28). In zeroth order we have (18). In first order assume the correction functions in the same form (19)-(20).

Substitution to (27) and limitation to first-order terms gives

$$
\begin{aligned}
& \eta_{t}+\eta_{x}+\alpha\left(a \eta_{y}+B_{x}+2 \eta \eta_{x}\right) \\
& +\varepsilon\left(C_{x}-\frac{1}{6} \eta_{3 x}-\frac{1}{3} \eta_{x 2 y}\right)=0 .
\end{aligned}
$$

Substitution to (28) and retention of terms up to first order gives

$$
\begin{aligned}
& \eta_{t}+\eta_{x}+\alpha\left(B_{t}+\eta \eta_{x}\right) \\
& \quad+\varepsilon\left(C_{t}-\frac{1}{2} \eta_{2 y t}-\frac{1}{2} \eta_{2 x t}\right)=0 .
\end{aligned}
$$

Substraction of (30) from (29) gives

$$
\begin{aligned}
& \alpha\left(B_{x}-B_{t}+\eta \eta_{x}+a \eta_{y}\right)+\varepsilon\left(C_{x}-C_{t}-\frac{1}{6} \eta_{3 x}\right. \\
& \left.-\frac{1}{3} \eta_{x 2 y}+\frac{1}{2} \eta_{2 y t}+\frac{1}{2} \eta_{2 x t}\right)=0 .
\end{aligned}
$$

In (31), we use the properties $B_{t}=-B_{x}, C_{t}=-C_{x}$, $\eta_{2 y t}=-\eta_{2 y x}, \eta_{2 x t}=-\eta_{3 x}$ valid in zeroth order. Since expressions in (31) are already in first order it is sufficient. Recall that the general form, e.g., $B_{t}=$ $-B_{x}+\alpha B a+\varepsilon B e$, and so on, do not change the further results since after insertion into (31) second-order terms have to be rejected. These properties and freedom of $\alpha, \varepsilon$ allow us to obtain simple differential equations for $B$ and $C$ in the form

$2 B_{x}+\eta \eta_{x}+a \eta_{y}=0, \quad 2 C_{x}-\frac{2}{3} \eta_{3 x}-\frac{5}{6} \eta_{x y y}=0$.

Integrating above equations one obtains

$$
\begin{gathered}
B=-\frac{1}{4} \eta^{2}-\frac{1}{2} a \int \eta_{y} d x, \\
\text { and } C=\frac{1}{3} \eta_{x x}+\frac{5}{12} \eta_{y y} .
\end{gathered}
$$

Then, the function $u$ becomes

$$
\begin{aligned}
u & =\eta+\alpha\left(-\frac{1}{4} \eta^{2}-\frac{1}{2} a \int \eta_{y} d x\right) \\
& +\varepsilon\left(\frac{1}{3} \eta_{x x}+\frac{5}{12} \eta_{y y}\right) .
\end{aligned}
$$

With $u$ in the form (33), both Boussinesq's equations should reduce to the same wave equation. Indeed, this is the case, and the final first-order wave equation receives the following form

$$
\begin{gathered}
\eta_{t}+\eta_{x}+\alpha\left(\frac{3}{2} \eta \eta_{x}+\frac{1}{2} a \eta_{y}\right) \\
+\varepsilon\left(\frac{1}{6} \eta_{x x x}+\frac{1}{12} \eta_{x y y}\right)=0 .
\end{gathered}
$$

Although the steps from assumed form of first-order functions (19)-(20) to equations (22) [1, Eq. (26)] or (34) are mathematically correct, the final equations are incorrect because the assumptions (19)-(20) violate the properties of the velocity potential. Details are explained in the next section.

\section{Critics}

The authors treat $u, v$ as independent functions, ignoring that, in fact, $u, v$ are partial derivatives of the same function $f$. Since $u=f_{x}$ and $v=f_{y}$, and these functions and their partial derivatives should be continuous, then the fundamental condition has to be fulfilled

$u_{y}=v_{x} \equiv f_{x y}$.

This condition is not fulfilled by the functions $u, v$ in equations (19)-(20) which are necessary for the authors to derive new $(2+1)$-dimensional equation. The requirement that condition (35) is fulfilled by (19)-(20) leads from one side to

$f_{x y} \equiv u_{y}=\eta_{y}+\alpha B_{y}+\varepsilon C_{y}$

and from the other side to

$f_{x y} \equiv v_{x}=0$.

So, the condition $u_{y}=v_{x}$ (35) can be fulfilled only when $\eta_{y}+\alpha B_{y}+\varepsilon C_{y}=0$ which, due to freedom of $\alpha$, $\varepsilon$, imply $B_{y}=C_{y}=\eta_{y}=0$ ! This means that the surface profile does not dependent on $y$ (there is translation symmetry in $y$-coordinate). In other words, searching for the solution in the form (19)-(20) reduces the problem to be only (1+1)-dimensional (with $\eta_{y}=0$ the final equation reduces to the usual $\mathrm{KdV}$ equation). Therefore, the derived first-order (2+1)-dimensional evolution equation cannot be the solution to the Boussinesq set (13)-(14). In the same way the equation (34) cannot be the solution to the correct Boussinesq's set (25)-(26). 
Can we extend the form of corrections (19)-(20) in such a way that they fulfill the condition (35)? We assume the most general form of first-order functions $u, v$, that is

$u=\eta+\alpha B(x, y, t)+\varepsilon C(x, y, t)$,

$v=a(x, t)+\alpha R(x, y, t)+\varepsilon S(x, y, t)$.

Inserting (36)-(37) into (27)-(28) and neglecting terms of second order one obtains

$$
\begin{aligned}
& \eta_{t}+\eta_{x}+\alpha\left(a \eta_{y}+B_{x}+2 \eta \eta_{x}+R_{y}\right) \\
& \quad+\varepsilon\left(C_{x}-\frac{1}{6} \eta_{x x x}-\frac{1}{6} \eta_{x y y}+S_{y}\right)=0
\end{aligned}
$$

and

$$
\begin{aligned}
& \eta_{t}+\eta_{x}+\alpha\left(B_{t}+\eta \eta_{x}+a a_{x}\right) \\
& \quad+\varepsilon\left(C_{t}-\frac{1}{2} \eta_{y y t}-\frac{1}{2} \eta_{x x t}\right)=0 .
\end{aligned}
$$

Substraction of (39) from (38) and use the properties $B_{t}=-B_{x}, \quad C_{t}=-C_{x}$ allows us to obtain two equations

$$
\begin{aligned}
& 2 B_{x}+\eta \eta_{x}-a a_{x}+a \eta_{y}+R_{y}=0, \quad \text { and } \\
& 2 C_{x}-\frac{2}{3}\left(\eta_{x x x}+\eta_{x y y}\right)+S_{y}=0 .
\end{aligned}
$$

Integration over $x$ gives

$$
\begin{aligned}
& B=-\frac{1}{4} \eta^{2}+\frac{1}{4} a^{2}-\frac{1}{2} a \int \eta_{y} d x-\frac{1}{2} \int R_{y} d x, \\
& C=\frac{1}{3}\left(\eta_{x x}+\eta_{y y}\right)-\frac{1}{2} \int S_{y} d x .
\end{aligned}
$$

So, in principle, the functions

$$
\begin{aligned}
& u=\eta+\alpha\left(-\frac{1}{4} \eta^{2}+\frac{1}{4} a^{2}-\frac{1}{2} a \int \eta_{y} d x-\frac{1}{2} \int R_{y} d x\right) \\
&+\varepsilon\left(\frac{1}{3}\left(\eta_{x x}+\eta_{y y}\right)-\frac{1}{2} \int S_{y} d x\right), \text { and } \\
& v=a(x, t)+\alpha R(x, y, t)+\varepsilon S(x, y, t),
\end{aligned}
$$

can make Boussinesq's equations compatible. But, is it possible to find such functions $a, R, S$ which ensure fulfilling the condition (35), that is ensure $u_{y}=v_{x}$ ? Because

$$
\begin{gathered}
u_{y}=\eta_{y}+\alpha\left(-\frac{1}{2} \eta \eta_{y}-\frac{1}{2} a \int \eta_{y y} d x-\frac{1}{2} \int R_{y y} d x\right) \\
+\varepsilon\left(\frac{1}{3}\left(\eta_{x x y}+\eta_{y y y}\right)-\frac{1}{2} \int S_{y y} d x\right)
\end{gathered}
$$

$v_{x}=a_{x}+\alpha R_{x}+\varepsilon S_{x}$,

this task seem to be hopeless.

\section{Another possibile approach}

The above considerations imply that in a shallow water problem, it is practically impossible to obtain $(2+1)$ dimensional evolution equation for the profile of surface wave $\eta(x, y, t)$, even in first-order approximation. However, this model can supply differential equations of any order for the function $f(x, y, t) \equiv \phi^{(0)}(x, y, t)$. Below, we present such an equation in first order.

Recall, that from (8) and the velocity potential (24) one obtains the first Boussinesq's equation in the form (25). The corresponding Boussinesq's equation resulting from (9) is

$\eta+f_{t}+\frac{1}{2} \alpha\left(f_{x}^{2}+f_{y}^{2}\right)-\frac{1}{2} \varepsilon\left(f_{x x t}+f_{y y t}\right)=0(47)$

Now, inserting

$\eta=-\left(f_{t}+\frac{1}{2} \alpha\left(f_{x}^{2}+f_{y}^{2}\right)-\frac{1}{2} \varepsilon\left(f_{x x t}+f_{y y t}\right)\right)$

from (47) into (25) and neglecting second-order terms one obtains first-order evolution equation for the function $f(x, y, t)$

$$
f_{x x}+f_{y y}-f_{t t}+\alpha\left[-f_{t}\left(f_{x x}+f_{y y}\right)-2\left(f_{x} f_{x t}+f_{y} f_{y t}\right)\right]
$$

$$
+\varepsilon\left[\frac{1}{2}\left(f_{x x t t}+f_{y y t t}\right)-\frac{1}{6}\left(f_{x x x x}+2 f_{x x y y}+f_{y y y y}\right)\right]=0 .
$$

It can be further simplified utilizing zeroth-order solution $f_{t t}=f_{x x}+f_{y y}$. Since the term $\varepsilon \frac{1}{2}\left(f_{x x t t}+f_{y y t t}\right)$ is already first order, we can use the equalities

$$
\begin{aligned}
& f_{x x t t}=\left(f_{t t}\right)_{x x}=\left(f_{x x}+f_{y y}\right)_{x x}=f_{x x x x}+f_{x x y y}, \\
& f_{y y t t}=\left(f_{t t}\right)_{y y}=\left(f_{x x}+f_{y y}\right)_{y y}=f_{x x y y}+f_{y y y y}
\end{aligned}
$$


to replace it by $\varepsilon \frac{1}{2}\left(f_{x x x x}+2 f_{x x y y}+f_{y y y y}\right)$. Therefore an equivalent, simpler form of (48), still valid up to first order, is

$$
\begin{aligned}
f_{x x} & +f_{y y}-f_{t t}+\alpha\left[-f_{t}\left(f_{x x}+f_{y y}\right)\right. \\
& \left.-2\left(f_{x} f_{x t}+f_{y} f_{y t}\right)\right] \\
& +\varepsilon\left(\frac{1}{3}\left(f_{x x x x}+2 f_{x x y y}+f_{y y y y}\right)\right)=0 .
\end{aligned}
$$

If the solution to (48) or (49) is known, the equation (47) supplies the surface profile function $\eta(x, y, t)$.

In general, for a flat bottom, one can extend this procedure to arbitrary order. However, even in first-order approximation partial differential equation (49) corresponding to $(2+1)$-dimensional shallow water problem is highly complicated. There is little hope to find either a solution to (49) or a (2+1)-dimensional wave equation for wave profile $\eta(x, y, t)$ without further significant simplifications.

\section{Conclusions}

We have proved that the (2+1)-dimensional KdV-type equation [1, Eq. (26)] has been inconsistently obtained by the authors and therefore cannot describe $(2+1)$ dimensional surface waves. Moreover, we have shown that when assumptions for first-order functions [1, Eqs. (14)-(15)] are used consistently with the properties of the velocity potential, then the solution reduces to the usual KdV equation. Additionally, we have demonstrated that even a consistent extension of the authors' method [1] gives no hope for obtaining appropriate first-order $(2+1)$-dimensional evolution equation for shallow water problem.

\section{Declarations}

Conflict of interest The authors declare that they have no conflict of interest.

Data availability No datasets were generated or analysed during the current study.

Preprint The preprint [4] has been deposited to arXiv and can be accessed at https://arxiv.org/abs/2105.08519.

Open Access This article is licensed under a Creative Commons Attribution 4.0 International License, which permits use, sharing, adaptation, distribution and reproduction in any medium or format, as long as you give appropriate credit to the original author(s) and the source, provide a link to the Creative Commons licence, and indicate if changes were made. The images or other third party material in this article are included in the article's Creative Commons licence, unless indicated otherwise in a credit line to the material. If material is not included in the article's Creative Commons licence and your intended use is not permitted by statutory regulation or exceeds the permitted use, you will need to obtain permission directly from the copyright holder. To view a copy of this licence, visit http://creativecommons.org/licenses/ by $/ 4.0 /$.

\section{References}

1. Fokou, M., Kofane, T.C., Mohamadou, A., Yomba, E.: Twodimensional third-and fifth-order nonlinear evolution equations for shallow water waves with surface tension. Nonlinear Dyn. 91, 1177-1189 (2018)

2. Burde, G.I., Sergyeyev, A.: Ordering of two small parameters in the shallow water wave problem. J. Phys. A: Math. Theor. 46, 075501 (2013)

3. Karczewska, A., Rozmej, P.: Can simple KdV-type equations be derived for shallow water problem with bottom bathymetry? Commun. Nonlinear Sci. Numer. Simulat. 82, 105073 (2020)

4. Rozmej, P., Karczewska, A.: Comment on "Two-dimensional third- and fifth- order nonlinear evolution equations for shallow water waves with surface tension" [Nonlinear Dyn, https://doi.org/10.1007/s11071-017-3938-7]https://arxiv. org/abs/2105.08519 (2021). [Preprint]

Publisher's Note Springer Nature remains neutral with regard to jurisdictional claims in published maps and institutional affiliations. 